\title{
Fully-Balanced Structures of Continuous-Time MLF OTA-C Filters
}

\author{
Yichuang Sun* and Kel Fidler** \\ * Department of Electrical and Electronic Engineering \\ University of Hertfordshire, Hatfield, Herts AL10 9AB, UK \\ Email: y.sun@herts.ac.uk, Tel: ++44 1707 284196, Fax: ++44 1707284199 \\ ** Department of Electronics, University of York, York YO1 5DD, UK
}

\begin{abstract}
This paper presents general fully balanced structures of continuous-time OTA-C filters based on multiple loop feedback (MLF) configurations. Both simple all pole and complex arbitrary zero fully balanced implementations are described. The general explicit iterative design formulae for the filters based on the important LF structure are presented. The design of fifth-order fully balanced LF filters is considered.
\end{abstract}

\section{Introduction}

Multiple loop feedback (MLF) active filters [1] have the advantage of both low sensitivity and arbitrary transmission zeros, compared with cascade structures that have high sensitivity and ladder topologies which can implement only imaginary axis zeros. Note that nonimaginary-axis zeros are required, for example, in equalizer design. OTA-C filters with multiple loop feedback configurations have received considerable attention in high frequency integrated continuous-time filter design [211]. Both integrators [2-8] and biquads [9-11] are used in the design. Integrator-based techniques may be most general, as biquads themselves are constructed based on integrators. This is especially true in integrated OTA-C filter design, because single-OTA biquads are no longer attractive as single-Opamp biquads in discrete Opamp-RC filter design. References [2, 3] have proposed integratorbased universal architectures using the IFLF structure with output summation and input distribution respectively. References $[4,5]$ have systematically explored the generation and design of integrator-based multiple loop feedback OTA-C filters with a new general unified design method having been developed and many new structures obtained including the LF configuration.

Note that fully balanced structures are most widely utilized in continuous-time integrated filter design, because balanced structures can achieve a very high commonmode rejection ratio and reduce both the even-order harmonic distortion components and the effects of power supply noise. Also, in practice, the LF feedback configuration is one of the most important multiple loop feedback structures, since it has very low sensitivity. In this paper, we hence propose a general method for generation of fully balanced multiple loop feedback OTA-C filters and give explicit design formulae for the filter design based on the LF feedback structure.

\section{General Fully-Balanced OTA-C Structures}

The general form of all-pole transfer functions can be expressed as

$$
H_{d}(s)=A_{0} /\left(B_{n} s^{n}+B_{n-1} s^{n-1}+\cdots+B_{1} s+1\right)
$$

The general single-ended all-pole multiple integrator loop feedback OTA-C model $[4,5]$ can be converted into the balanced equivalent by using differential four input and two output OTAs (for example, the one in [6]) in integrators and mirroring the feedback network in the upper part to the lower part, as shown in figure 1. With $\tau_{j}=C_{j} / g_{m j}$ denoting the voltage integration constant of the $j$ th integrator and $f_{i j}$ the voltage feedback coefficient from the output of the $j$ th integrator to the negative input terminal of the $i$ th integrator, the transfer function can be derived as

$$
H(s)=\frac{V_{\text {out }}}{V_{\text {int }}}=1 /|A(s)|
$$

where $|A(s)|$ is the determinant of system coefficient ma$\operatorname{trix} A(s)$ of

$$
\left[\begin{array}{ccccc}
s \tau_{1}+f_{11} & f_{12} & f_{13} & & f_{1 n} \\
-1 & s \tau_{2}+f_{22} & f_{23} & & f_{2 n} \\
& -1 & s \tau_{3}+f_{33} & & f_{3 n} \\
& & & \cdots & \\
& & & & s \tau_{n}+f_{n n}
\end{array}\right]
$$

The feedback coefficent matrix $F=\left[f_{i j}\right]$ has the property that $f_{i j} \neq 0$ if there is feedback between the negative input terminal of integrator $i$ and the output of integrator $j$; and otherwise, it is zero. The nonzero feedback coefficient can always be realized using an OTA voltage 
amplifier and the zero feedback coefficient can be obtained simply by an open circuit. The unity feedback coefficient can also be achieved by direct wire connection. If all the nonzero feedback coefficients are unity and are realized with pure wire connection, the whole system then has the minimum number of components.

Similarly, we consider fully balanced OTA-C structures for the synthesis of the universal transfer function

$$
H_{d}(s)=\frac{A_{n} s^{n}+A_{n-1} s^{n-1}+\cdots+A_{1} s+A_{0}}{B_{n} s^{n}+B_{n-1} s^{n-1}+\cdots+B_{1} s+1}
$$

The first general fully balanced OTA-C model for implementing arbitrary filter characteristics is shown in figure 2. As depicted, this model is composed of a multiple loop feedback OTA-C network and an output summation OTA network. Denoting $k_{j}=g_{a j} / g_{r}$, we clerive the circuit transfer function

$$
H(s)=\frac{V_{\text {out }}}{V_{\text {int }}}=k_{0}+\frac{1}{|A(s)|} \sum_{j=1}^{n} k_{j} A_{1 j}(s)
$$

where $A_{i j}(s)$ represent the cofactors of matix $A(s)$. The system poles are determined by $\tau_{j}$ and $f_{i j}$ and the transmission zeros may be controlled arbitrarily by transconductances $g_{a j}$ through weights $k_{j}$.

The second fully balanced structures for implementation of finite transmission zeros is shown in figure 3 . In this configuration the voltage signal is applied to circuit nodes by an input distribution OTA network. With $\beta_{j}=g_{a j} / g_{m j}$ and $\gamma=g_{n \ell} / g_{r}$, we can formulate

$$
H(s)=\frac{V_{\text {out }}}{V_{\text {in }}}=\gamma\left[\beta_{0}+\frac{1}{|A(s)|} \sum_{j=1}^{n} \beta_{j} A_{j n}(s)\right]
$$

Again, any filter transmission characteristics may be realized through adjusting distribution weights $\beta_{j}$, that is, the associated $g_{a j}$.

Note that if the maximum order in the numerator is required to be $n-1$, then we can remove the $g_{a 0}$ OTA, and the $g_{m 0}$ and $g_{r}$ OTAs for $\gamma=1$ and simply output the voltage $V_{o n}$ directly in the distribution case (this leads to an advantage that the resistive summing node that will have effects of the parasitics at very high frequencies is avoided), while for the summation the $g_{a 0}$ OTA should be deleted. It is also of interest to note that when the transadmittance functions are required, we can eliminate the $g_{r}$ OTA in both the input distribution and ouput sumnation configurations.

\section{Fully-Balanced LF Structures and Design Formulae}

The design methods and formulae discussed systematically in $[4,5]$ are completely suitable for the design of fully balanced structures presented in the above. We now further give the design formulae for the important LF feedback structure that corresponds to $f_{i j}=1$ for $j=i+1, i=1,2, \cdots n-1$ and $f_{n n}=1$, and for the other $i, j, f_{i j}=0$.

For the distribution form, $A_{j n}$ can be formulated in an iterative way as

$$
\begin{gathered}
A_{1 n}(s)=1, \quad A_{2 n}(s)=\tau_{1} s, \\
A_{j n}(s)=s \tau_{j-1} A_{(j-1) n}(s)+A_{(j-2) n}(s)
\end{gathered}
$$

where $j=3,4,5, \cdots, n$.

For the summation type we determine $A_{1 j}(s)$ using

$$
\begin{aligned}
& A_{1 n}(s)=1, A_{1(n-1)}(s)=s \tau_{n}+1, \\
& A_{1 j}(s)=s \tau_{j+1} A_{1(j+1)}(s)+A_{1(j+2)}
\end{aligned}
$$

where $j=n-2, n-3, n-4, \cdots, 1$.

$|A(s)|$ can be obtained by

$|A(s)|=\left(s \tau_{n}+1\right) A_{n n}(s)+A_{(n-1) n}(s)=s \tau_{1} A_{11}(s)+A_{12}(s)$

For any order, using the above iterative formulae and equations (2), (5) and (6) we can derive the corresponding transfer function $H(s)=V_{\text {out }} / V_{\text {in }}=N(s) / D(s)$. Take the fifth-order as an example. In the all-pole case, using (2) and (9) we have $N(s)=1$ and

$D(s)=\tau_{1} \tau_{2} \tau_{3} \tau_{4} \tau_{5} s^{5}+\tau_{1} \tau_{2} \tau_{3} \tau_{4} s^{4}+\left(\tau_{1} \tau_{2} \tau_{3}+\tau_{1} \tau_{2} \tau_{5}+\tau_{1} \tau_{4} \tau_{5}\right.$
$\left.+\tau_{3} \tau_{4} \tau_{5}\right) s^{3}+\left(\tau_{1} \tau_{2}+\tau_{1} \tau_{4}+\tau_{3} \tau_{4}\right) s^{2}+\left(\tau_{1}+\tau_{3}+\tau_{5}\right) s+1$

For the input distribution structure, using (6) and (7) we have the numerator of the transfer function as $(\gamma=1)$

$$
N(s)=\beta_{0} \tau_{1} \tau_{2} \tau_{3} \tau_{4} \tau_{5} s^{5}+\left(\beta_{0}+\beta_{5}\right) \tau_{1} \tau_{2} \tau_{3} \tau_{4} s^{4}
$$

$+\left[\beta_{0}\left(\tau_{1} \tau_{2} \tau_{3}+\tau_{1} \tau_{2} \tau_{5}+\tau_{1} \tau_{4} \tau_{5}+\tau_{3} \tau_{4} \tau_{5}\right)+\beta_{4} \tau_{1} \tau_{2} \tau_{3}\right] s^{3}$

$+\left[\left(\beta_{0}+\beta_{5}\right)\left(\tau_{1} \tau_{2}+\tau_{1} \tau_{4}+\tau_{3} \tau_{4}\right)+\beta_{3} \tau_{1} \tau_{2}\right] s^{2}+\left[\beta_{0}\left(\tau_{1}+\tau_{3}+\tau_{5}\right)\right.$

$$
\left.\left.+\beta_{4}\left(\tau_{1}+\tau_{3}\right)+\beta_{2} \tau_{1}\right)\right] s+\left(\beta_{0}+\beta_{5}+\beta_{3}+\beta_{1}\right)
$$

For the output summation type, the numerator of the transfer function is formulated using (5) and (8) as

$$
\begin{gathered}
N(s)=k_{0} \tau_{1} \tau_{2} \tau_{3} \tau_{4} \tau_{5} s^{5}+\left(k_{0} \tau_{1} \tau_{2} \tau_{3} \tau_{4}+k_{1} \tau_{2} \tau_{3} \tau_{4} \tau_{5}\right) s^{4}+ \\
{\left[k_{0}\left(\tau_{1} \tau_{2} \tau_{3}+\tau_{1} \tau_{2} \tau_{5}+\tau_{1} \tau_{4} \tau_{5}+\tau_{3} \tau_{4} \tau_{5}\right)+k_{1} \tau_{2} \tau_{3} \tau_{4}+k_{2} \tau_{3} \tau_{4} \tau_{5}\right] s^{3}} \\
+\left[k_{0}\left(\tau_{1} \tau_{2}+\tau_{1} \tau_{4}+\tau_{3} \tau_{4}\right)+k_{1}\left(\tau_{2} \tau_{3}+\tau_{2} \tau_{5}+\tau_{4} \tau_{5}\right)+k_{2} \tau_{3} \tau_{4}\right. \\
\left.+k_{3} \tau_{4} \tau_{5}\right] s^{2}+\left[k_{0}\left(\tau_{1}+\tau_{3}+\tau_{5}\right)+k_{1}\left(\tau_{2}+\tau_{4}\right)+k_{2}\left(\tau_{3}+\tau_{5}\right)\right. \\
\left.+k_{3} \tau_{4}+k_{4} \tau_{5}\right] s+\left(k_{0}+k_{1}+k_{2}+k_{3}+k_{4}+k_{5}\right)
\end{gathered}
$$

To realize the fifth-order transfer function in (4), the pole parameters, $\tau_{j}$ in the denominator of the transfer function in (10) are determined as

$$
\tau_{5}=\frac{B_{5}}{B_{4}}, \tau_{4}=\frac{B_{4}}{B_{3}-B_{2} \tau_{5}}, \tau_{3}=\frac{B_{3}-B_{2} \tau_{5}}{B_{2}-\left(B_{1}-\tau_{5}\right) \tau_{4}},
$$




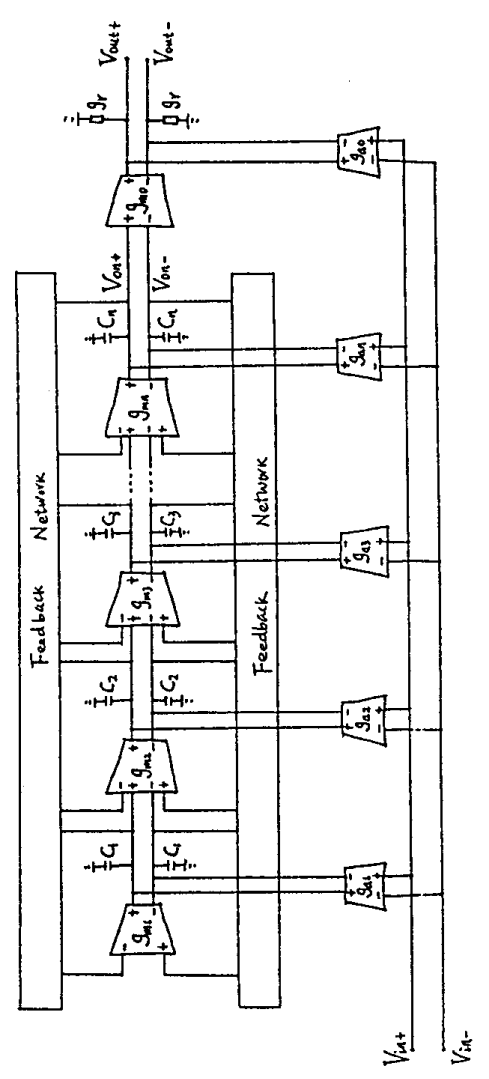

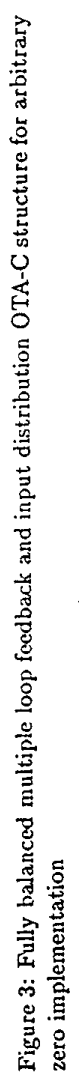
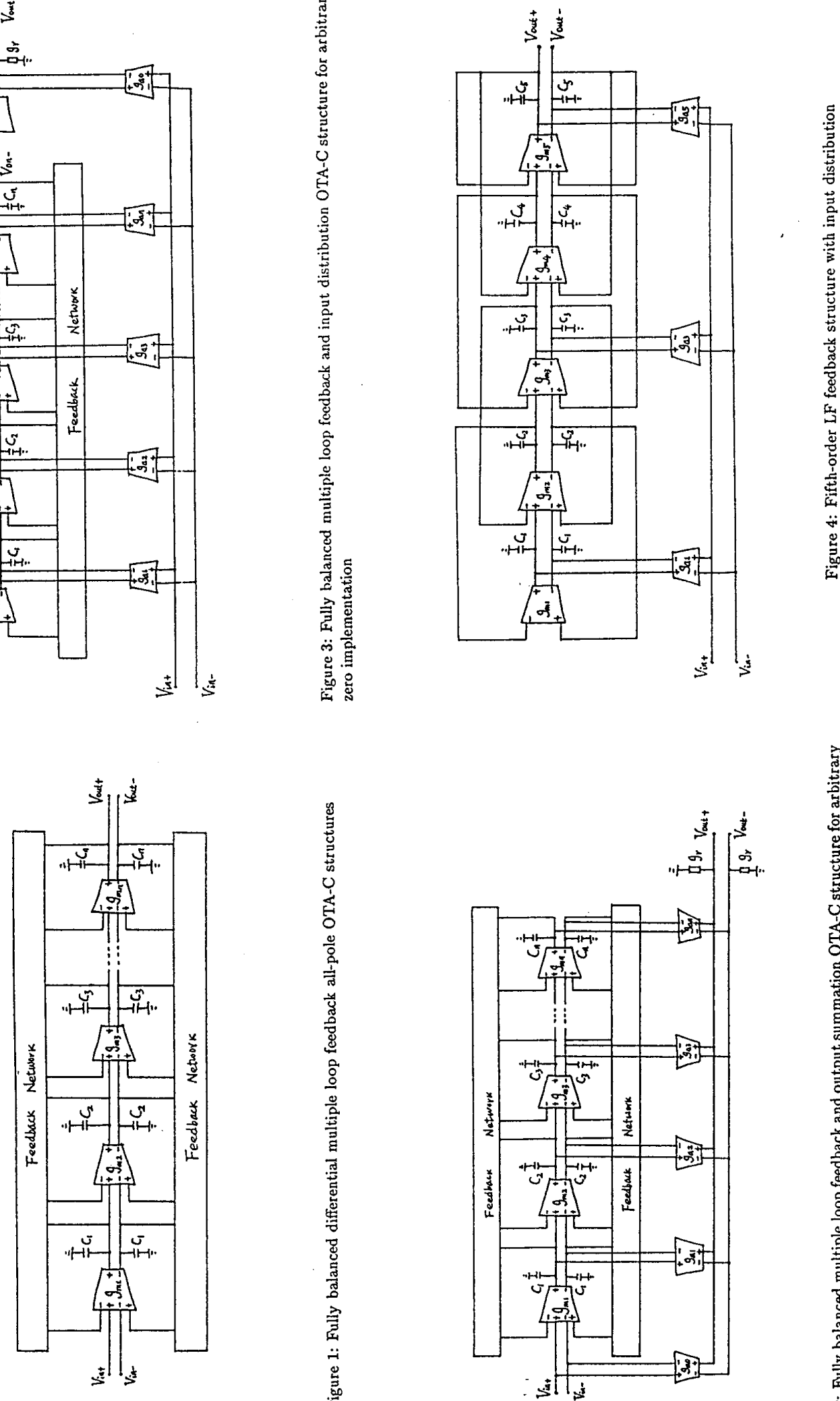

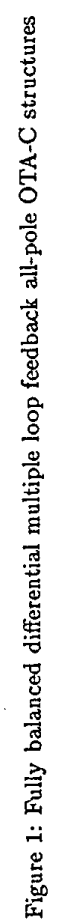

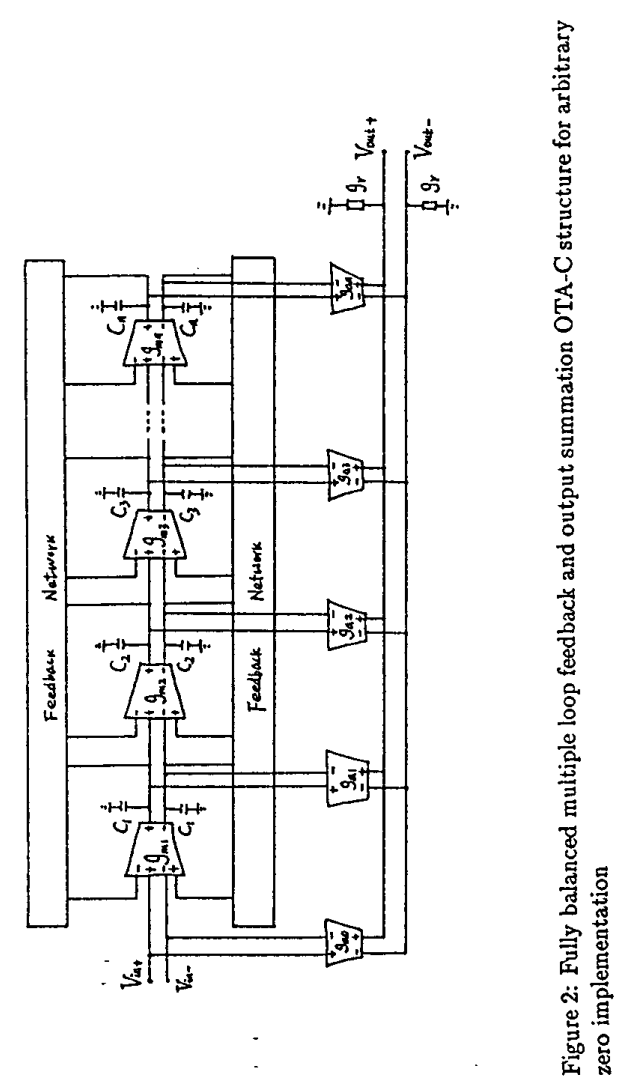




$$
\tau_{2}=\frac{B_{2}-\left(B_{1}-\tau_{5}\right) \tau_{4}}{B_{1}-\tau_{3}-\tau_{5}}, \tau_{1}=B_{1}-\tau_{3}-\tau_{5}
$$

The numerator parameters, $\beta_{j}$ for the distribution type and $k_{j}$ for the summation can be easily determined from (11) and (12) with comparison with (4) in an iterative way respectively, since $\tau_{j}$ are determined by (13). Figure 4 shows a fifth-order low-pass filter realizing the numerator coefficients of $A_{j}=0, \quad j=1,3,5$, which is obtainable from figure 3 by removing the $g_{a 0}$ OTA $\left(\beta_{0}=0\right)$, replacing the $g_{m 0}$ and $g_{r}$ OTA's by a direct connection, and removing the $g_{a 2}$ and $g_{a 4}$ OTAs $\left(\beta_{2}=\beta_{4}=0\right)$. The numerator parameters can be determined as $\beta_{5}=$ $A_{4} / B_{4}, \beta_{3}=\left(A_{2}-\beta_{5} B_{2}\right) / \tau_{1} \tau_{2}, \beta_{1}=A_{0}-\left(\beta_{3}+\beta_{5}\right)$.

It can be shown that for any order $n$ in any case of all-pole and arbitrary zero realizations, the parameters, $\tau_{j}, k_{j}$ or $\beta_{j}$ all can be determined explicitly. This will be discussed separately.

\section{Conclusions}

General fully balanced multiple loop feedback OTA-C filter structures have been constructed based on the singleended counterparts previously proposed by the authors. The design formulae for the low sensitivity LF feedback structure have also been derived. The results in the paper can also be extended for the design of current-mode multiple loop feedback OTA-C filters $[7,8]$.

\section{References}

[1] K. R. Laker, R. Schaumann, and M. S. Ghausi, "Multiple-loop feedback topologies for the design of lowsensitivity active filters," IEEE Trans. Circuits Syst., Vol.26, pp.1-21, 1979.

[2] Y. Sun and J. K. Fidler, "OTA-C realization of general high-order transfer functions," Electronics Letters, Vol.29, No.12, pp.1057-1058, 1993.

[3] Y. Sun and J. K. Fidler, "Synthesis and performance analysis of a universal minimum component integratorbased IFLF OTA-grounded capacitor filter," IEE Proceedings: Circuits, Devices and Systems, Vol.143, pp.107-114, 1996.

[4] Y. Sun and J. K. Fidler, "Minimum component multiple integrator loop OTA-grounded capacitor all-pole filters," Proc. IEEE Midwest Symp. on Circuits and Systems, pp.983-986, Lafayette, USA, 1994.

[5] Y. Sun and J. K. Fidler, "Structure generation and design of multiple loop feedback OTA-grounded capacitor filters," IEEE Trans. Circuits Syst., Part-I, Vol.44, No.1, 1997.

[6] D. H. Chiang and R. Schaumann, "A CMOS fullybalanced continuous time IFLF filter design for $\mathrm{read} /$ wire channels," IEEE Int. Symp. Circuits Syst, Vol.1, pp.167-170, 1996.
[7] Y. Sun and J. K. Fidler, "Current-mode multiple loop feedback filters using dual output OTAs and grounded capacitors," Int. J. Circuit Theory and Applications, vol.25, pp.69-80, 1997.

[8] Y. Sun and J. K. Fidler, "Current-mode OTA-C realisation of arbitrary filter characteristics," Electronics Letters, Vol.32, pp.1181-1182, 1996.

[9] R. Nawrocki, "Electronically controlled OTA-C filter with follow-the-leader-feedback structures," Int. J. Circuit Theory and Applications, Vol.16, pp.93-96, 1988.

[10] F. Krummenacher, "Design considerations in highfrequency CMOS transconductance amplifier capacitor (TAC) filters," Proc. IEEE Int. Symp. Circuits Syst., pp.100-105, 1989.

[11] A. N. Gonuleren, R. Kopru and H. Kuntman, "Multiloop feedback bandpass OTA-C filters using quads," Proc. Euro. Conf. Circuit Theory and Design, Vol.2, pp.607-610, Istanbul, 1995. 